\title{
Anorexia nervosa of the restrictive type and celiac disease in adolescence
}

\author{
This article was published in the following Dove Press journal: \\ Neuropsychiatric Disease and Treatment \\ 4 May 2017 \\ Number of times this article has been viewed
}

\author{
Renata Nacinovich' \\ Lucio Tremolizzo ${ }^{2}$ \\ Fabiola Corbetta' \\ Elisa Conti ${ }^{2}$ \\ Francesca Neri ${ }^{1}$ \\ Monica Bomba' \\ 'Child and Adolescent Mental Health \\ Department, ${ }^{2}$ Neurology Unit and \\ Lab of Neurobiology, San Gerardo \\ Hospital, School of Medicine and \\ Surgery and Milan Center for \\ Neuroscience (Neuro-MI), University \\ of Milano-Bicocca, Monza, Italy
}

Background: Anorexia nervosa (AN) is usually present in adolescence with symptoms partially overlapping celiac disease (CD), but the relationship between these two conditions has received little attention in the literature. The aim of this work was to explore this relationship, considering if $\mathrm{CD}$ could be associated with specific baseline AN-related clinical features.

Methods: In this retrospective study, 82 adolescent female out- and inpatients with AN of the restrictive type (ANr), according to the Diagnostic and Statistical Manual of Mental Disorders, Fifth Edition criteria, were recruited. CD diagnosis and related serology were recorded, including tissue transglutaminase type- 2 antibodies, endomysial antibodies, and antibodies against deamidated forms of gliadin peptides. Eating disorder inventory-3, Children's Depression Inventory, body mass index, age, and disease duration data recorded at the time of blood withdrawal were also obtained from each patient.

Results: Five (6.1\%) subjects presented a CD disorder associated with AN: none of the collected psychometric measures was significantly correlated with any CD-related parameter or characterized as a specific subgroup.

Conclusion: CD diagnosis or serology does not relate to ANr clinical or demographic characteristics. However, a slight increase in prevalence with respect to the general population might be hypothesized and possibly elucidated by further studies with an appropriate design.

Keywords: anorexia nervosa, celiac disease, adolescence, celiac disease antibody, gluten

\section{Introduction}

Celiac disease (CD) is a common autoimmune disorder characterized by gluten-induced systemic symptoms and signs, presence of specific antibodies and specific human leukocyte antigen type, and characteristic histological changes in the duodenum. At the onset, patients may present typical manifestation (diarrhea, weight loss), may be oligosymptomatic, or may present atypical symptoms such as headache and psychiatric disturbances, ${ }^{1}$ including eating disorders (EDs). ${ }^{2}$ Anorexia nervosa (AN) is one of the most common psychiatric diagnoses in adolescent girls $(<1 \%-4 \%$ of the female population). It is a serious, potentially life-threatening ED characterized by self-starvation, with important weight loss associated with an intense fear of weight gain and persistent behavior to prevent weight gain and by self-esteem overly related to body image. ${ }^{3}$ AN presents usually in the adolescence with symptoms partially overlapping CD (in particular, weight loss and psychiatric disorders), but the relationship between these two conditions has received little attention in the literature, ${ }^{4}$ with few case reports and small case series published, ${ }^{4,5}$ again often in adult patients. ${ }^{6}$ Only recently, Basso et $\mathrm{al}^{7}$ reported the prevalence of $\mathrm{CD}$ in a population of 177 adolescents affected by AN (males and females, restrictive and binge/purging type) and found that the overall prevalence of $\mathrm{CD}$ in their sample was the same as that in the general
Correspondence: Renata Nacinovich Child and Adolescent Mental Health Department, San Gerardo Hospital, University of Milano Bicocca, via Pergolesi 33, 20900 Monza (MB), Italy

Tel +39039233 3579

Fax +390392333321

Email renata.nacinovich@unimib.it
Neuropsychiatric Disease and Treatment 2017:13 1211-1214

| 2 | | 
population (1:100/200). ${ }^{7}$ They concluded that AN patients may not require regular screening for $\mathrm{CD}$. On the other hand, a recent meta-analysis highlighted the greater presence $(5.3 \%-44.4 \%)$ of EDs in population with gastrointestinal disorders, including CD. ${ }^{8}$

Undiagnosed CD predisposes to mental and behavioral disorders, and great attention should be maintained to exclude this illness in subjects with AN. ${ }^{7}$ Moreover, the association with $\mathrm{CD}$ might represent a biological and psychological prognostic risk factor for subjects affected by AN of the restrictive type (Anr), since the two conditions might mutually reinforce their clinical expression.

The main objective of this study was to assess whether the prevalence of CD in adolescents with ANr is higher than that reported in the general population, requiring a regular screening program. Moreover, another aim of this study was to determine a possible association between CD and baseline ANr-related clinical features.

\section{Materials and methods}

Eighty-two adolescent female outpatients and inpatients consecutively accessing to the Eating Disorder Service of the Child and Adolescent Mental Health Department of Monza (Italy) were enrolled. All the subjects were diagnosed with ANr according to the Diagnostic and Statistical Manual of Mental Disorders, Fifth Edition criteria. CD-specific serological tests were performed. In particular, tissue transglutaminase type-2 antibodies (anti-TG2), endomysial antibodies (EMA), and antibodies against deamidated forms of gliadin peptides (DGP) were analyzed to assess CD. If any total antibody determination was over the laboratory cutoff, a second determination distinguishing IgA and IgG subtypes was performed. IgA deficiency was investigated as well according to guidelines. ${ }^{9,10}$ Moreover, to assess the specific ED features and the presence of depressive traits at the time of blood withdrawal, patients completed the Italianvalidated version of Eating Disorder Inventory- $3^{11}$ and of the Children's Depression Inventory. ${ }^{12}$ The research was approved by the institutional review board of A.S.S.T. Monza (Healthcare System of Regione Lombardia - San Gerardo Hospital). All subjects and their parents were asked to sign an informed consent to participate in the study.

\section{Statistical analysis}

Continuous variables were expressed as mean \pm standard deviation (SD), whereas categorical variables were expressed as percentages. Analysis of variance was used for the analysis of continuous variables, and the nonparametric Spearman test was used for the analysis of correlations. The level of significance was set at $P<0.05$. Analysis was completed using Statistical Package for the Social Sciences statistical package (Version 21.0; IBM Corporation, Armonk, NY, USA).

\section{Results}

Clinical and demographic data of the recruited sample are shown in Table 1 . Three patients (3.7\%) of our series (AN CD+) were already diagnosed with a biopsy-proven CD before arriving to our attention for the ANr diagnosis and were under a gluten-free diet; for this reason, we reported them separately. Anti-TG2 serology allowed the identification of two patients $(2.4 \%)$ who were eventually diagnosed with $\mathrm{CD}$ following a duodenal biopsy $(\mathrm{AN}+)$. Eight additional patients $(9.8 \%)$ presented with anti-DGP antibodies above cutoff values (AN- DGP+). None of them presented anti-EMA and, at the time, no further investigations were performed. In the remaining 69 cases $(84.1 \%, A N-), C D$ serology was not significant. Neither age, disease duration, body mass index (BMI), Eating Disorder Inventory-3-specific subscores, and composites nor Children's Depression Inventory was significantly associated with any CD measure or characterized as a specific group.

\section{Discussion}

The results of the present study suggest that CD serology might not be useless in Eating Disorders Services as a screening strategy in ANr for detecting mimics or comorbidities even if our figures are higher than those expected in the general population $(2.4 \%$ new diagnosis vs $1 \%){ }^{7}$ As suggested by Urban-Kowalczy, ${ }^{13}$ the screening for CD would be recommended in AN patients in which the symptoms, such as digestive complaints, are only partially responding to psychiatric interventions. We sought to identify those AN patients carrying any CD-antibody positivity, besides a full CD diagnosis, since many psychiatric conditions, including EDs, ${ }^{2}$ have been variably associated with the presence of these antibodies, as well as diffuse brain changes. ${ }^{14}$ None of the predetermined clinical characteristics could appreciably distinguish $\mathrm{AN}-$ patients from AN- DGP+ ones; furthermore, the five CD-diagnosed AN adolescents did not clinically differ in a significant manner from the other recruited patients. Nevertheless, different from data obtained by Basso et $\mathrm{al}^{7}$ in a more heterogeneous sample of adolescents with EDs, our results suggest the presence of a slight clinical overlap with $\mathrm{CD}$ in the presentation of $\mathrm{ANr}$ in female adolescent patients. In a clinical perspective, the comorbidity of CD might increase the medical complications 
Table I Clinical characteristics of the recruited sample

\begin{tabular}{|c|c|c|c|c|}
\hline Variables & $\mathbf{A N}-$ & $\mathbf{A N}+$ & AN CD+ & AN- DGP+ \\
\hline Patients, n (\%) & $69(84.1)$ & $2(2.4)$ & $3(3.7)$ & $8(9.8)$ \\
\hline Age, years & $14.5 \pm 1.4(11-17)$ & $13-15$ & $12-13-16$ & $14.8 \pm 1(13-16)$ \\
\hline Duration, months & $10.5 \pm 9.7(1-42)$ & $20-5$ & $6-5-7$ & $6.4 \pm 5.4(2-18)$ \\
\hline $\mathrm{BMI}, \mathrm{kg} / \mathrm{m}^{2}$ & $15.0 \pm 1.8(10.7-19.7)$ & $13.5-15.4$ & $\mid 4.4-14.5-16.7$ & $15.8 \pm 2.4(\mid 3.2-19.3)$ \\
\hline EDI3-DT & $64.8 \pm 26.5(0-99)$ & $80-21$ & 68 & $57 \pm 17.3(27-75)$ \\
\hline EDI3-B & $32.8 \pm 32.0(0-96)$ & $0-15$ & $0-0-0$ & $28.6 \pm 34.9(0-83)$ \\
\hline EDI3-BD & $59.4 \pm 25.7$ (I7-99) & $56-35$ & $8-17-12$ & $49.5 \pm 24.7(14-94)$ \\
\hline EDI3-EDRC & $60.4 \pm 24.4(7-95)$ & $67-7 \mid$ & $34-73-85$ & $51.9 \pm 21.2(13-77)$ \\
\hline EDI3-GPMC & $59.1 \pm 23.1$ (8-99) & $|8-10|$ & $67-73-98$ & $60.5 \pm 23.5(25-85)$ \\
\hline $\mathrm{CDI} \geq 20, \mathrm{n}(\%)$ & $15(21.7)$ & Score: $5-26$ & $20-|7-1|$ & $2(25)$ \\
\hline anti-TG2 tot $(\mathrm{NV}<15 \mathrm{U} / \mathrm{mL})$ & $2.8 \pm 2.0(1-13.7)$ & $62.0-304.7$ & $3.1-7.0-6.8$ & $2.8 \pm 1.2(1.2-4.7)$ \\
\hline anti-TG2 IgA & NP & 83.9-288.3 & NP & NP \\
\hline anti-TG2 IgG & NP & $11.4-10.1$ & NP & NP \\
\hline anti-EMA & Neg & Pos-pos & Neg-neg-neg & Neg \\
\hline anti-DGP tot (NV < I5 U/mL) & $6.8 \pm 3.2(1.9-14.3)$ & $39.2-152.1$ & $5.23-4.4-6.1$ & $29.5 \pm 6.7(21.6-4 I)$ \\
\hline anti-DGP IgA & NP & $5.7-37.8$ & NP & $4.8 \pm 2.8(2.1-10.5)$ \\
\hline anti-DGP IgG & NP & $32.2->100$ & NP & $40.8 \pm 22.2(10.4-66.1)$ \\
\hline IgA tot (NV: 6I-348 mg/dL) & $155.4 \pm 66(0-37 \mid)$ & $103-172$ & $125-195-99$ & $189 \pm 95.9(77-333)$ \\
\hline IgA deficiency, n (\%) & $2(3.1)$ & Neg-neg & Neg-neg & Neg \\
\hline Duodenal biopsy & NP & Pos-pos & Pos-pos & NP \\
\hline
\end{tabular}

Note: Continuous data are reported as mean \pm standard deviation (range).

Abbreviations: AN, anorexia nervosa; BMI, body mass index; CD, celiac disease; CDI, Children's Depression Inventory; DGP, deamidated forms of gliadin peptides; EDI3, Eating Disorder Inventory-3; -B, bulimia; -BD, body dissatisfaction; -DT, drive for thinness; -EDRC, eating disorder risk composite; -GPMC, general psychological maladjustment composite; EMA, endomysial antibodies; NP, not performed; NV, normal values; TG2, tissue transglutaminase type-2; pos, positive; neg, negative; tot, total.

of $\mathrm{ANr} .^{3}$ Moreover, alimentation and digestion in adolescents with $\mathrm{ANr}$ and $\mathrm{CD}$ might represent a biological and psychological locus minoris resistentiae (ie, weakness), from both a biological and psychological point of view. $\mathrm{ANr}$ and CD could mutually reinforce their clinical expression: as it occurs in other somatic conditions, such as weight control through insulin underdosing in anorexic subjects with type 1 diabetes, ${ }^{3}$ we observed the deliberate gluten intake as a weight-loss strategy in one case of celiac subjects with ANr. Certainly, the major caveat is that our report is underpowered for detecting a meaningful difference in this sense and the research should be repeated with an adequate number of subjects. Even Ricca et $\mathrm{l}^{15}$ stressed the importance of future investigations because of the clinical significance and prognosis of $\mathrm{AN}$, together with the possible diagnostic problems related to the similarity of many of the symptoms that characterize AN and CD. Moreover, we studied adolescents with ANr, but we did not exclude the opportunity of analyzing a possible relationship between CD and other EDs. Possibly, other presentations such as nonceliac gluten sensitivity might be considered for future investigations. ${ }^{16}$

\section{Disclosure}

The authors report no conflicts of interest in this work.

\section{References}

1. Bern EM, O'Brien RF. Is it an eating disorder, gastrointestinal disorder, or both? Curr Opin Pediatr. 2013;25(4):463-470.

2. Passananti V, Siniscalchi M, Zingone F, et al. Prevalence of eating disorders in adults with celiac disease. Gastroenterol Res Pract. 2013;2013: 491657-491662.

3. Keski-Rahkonen A, Mustelin L. Epidemiology of eating disorders in Europe: prevalence, incidence, comorbidity, course, consequences, and risk factors. Curr Opin Psychiatry. 2016;29(6):340-345.

4. Mascitelli L, Pezzetta F, Goldstein MR. Coeliac disease and anorexia nervosa. Intern Med J. 2009;39(11):784.

5. Leffler DA, Dennis M, Edwards George JB, Kelly CP. The interaction between eating disorders and celiac disease: an exploration of 10 cases. Eur J Gastroenterol Hepatol. 2007;19(3):251-255.

6. Yucel B, Ozbey N, Demir K, Polat A, Yager J. Eating disorders and celiac disease: a case report. Int J Eat Disord. 2006;39(6):530-532.

7. Basso MS, Zanna V, Panetta F, et al. Is the screening for celiac disease useful in anorexia nervosa? Eur J Pediatr. 2013;172(2): 261-263.

8. Greco L, Timpone L, Abkari A, et al. Burden of celiac disease in the Mediterranean area. World J Gastroenterol. 2011;17(45): 4971-4978.

9. Satherley R, Howard R, Higgs S. Disordered eating practices in gastrointestinal disorders. Appetite. 2015;84:240-250.

10. Husby S, Koletzko S, Korponay-Szabó IR, et al; ESPGHAN Working Group on Coeliac Disease Diagnosis; ESPGHAN Gastroenterology Committee; European Society for Pediatric Gastroenterology, Hepatology, and Nutrition. European Society for Pediatric Gastroenterology, Hepatology, and Nutrition guidelines for the diagnosis of coeliac disease. J Pediatr Gastroenterol Nutr. 2012;54:136-160.

11. Garner DM. Italian Version. In: Giannini M, Pannocchia L, Dalle Grave R, Muratori e Valentina Viglione F, editors. Eating Disorder Inventory-3. Firenze: Organizzazioni Speciali; 2008. 
12. Kovacs M. Italian adaptation. In: Camuffo M, Cerutti R, Lucarelli L, Mayer R, editors. C.D.I.: Children's Depression Inventory, Questionario di autovalutazione. Firenze: Organizzazioni Speciali; 1988.

13. Urban-Kowalczy M. Psychiatric complications of celiac disease. Int $J$ Celiac Dis. 2015;3(1):25-27.

14. Bilgic B, Aygun D, Arslan AB, et al. Silent neurological involvement in biopsy-defined coeliac patients. Neurol Sci. 2013;34(12):2199-2204.
15. Ricca V, Mannucci E, Calabrò A, Bernardo MD, Cabras PL, Rotella CM. Anorexia nervosa and celiac disease: two case reports. Int J Eat Disord. 2000;27(1):119-122.

16. Volta U, Bardella MT, Calabrò A, Troncone R, Corazza GR; Study Group for Non-Celiac Gluten Sensitivity. An Italian prospective multicenter survey on patients suspected of having non-celiac gluten sensitivity. BMC Med. 2014;12:85-92.

\section{Publish your work in this journal}

Neuropsychiatric Disease and Treatment is an international, peerreviewed journal of clinical therapeutics and pharmacology focusing on concise rapid reporting of clinical or pre-clinical studies on a range of neuropsychiatric and neurological disorders. This journal is indexed on PubMed Central, the 'PsycINFO' database and CAS, and is the official journal of The International Neuropsychiatric Association (INA). The manuscript management system is completely online and includes a very quick and fair peer-review system, which is all easy to use. Visit http://www.dovepress.com/testimonials.php to read real quotes from published authors.

\footnotetext{
Submit your manuscript here: http://www.dovepress.com/neuropsychiatric-disease-and-treatment-journal
} 